\title{
Asymptotic Critical Transmission Ranges for Connectivity in Wireless Ad Hoc Networks with Bernoulli Nodes
}

\author{
Peng-Jun Wan Chih-Wei Yi \\ Department of Computer Science \\ Illinois Institute of Technology \\ 10 West 31st Street, Chicago, IL 60616, USA \\ Email:wan@cs.iit.edu_yichihw@iit.edu
}

\begin{abstract}
Wireless ad hoc networks with Bernoulli nodes provide a unified model of various important problems including fault-tolerance, randomized construction of virtual backbone, randomized broadcast routing, and randomized wake/sleep management. We assume that the wireless ad hoc network consists of $n$ nodes which are distributed independently and uniformly in a unit-area disk and are active (or available) independently with some constant probability $p$. Let $\rho_{n}$ denote the random variable which is the smallest transmission range at which the active nodes form a connected network, and $\rho_{n}^{\prime}$ denote the random variable which is the smallest transmission range at which the active nodes form a connected network and each inactive node is adjacent to at least one active node. $\rho_{n}$ is referred to as the critical transmission range for connectivity of active nodes, and $\rho_{n}^{\prime}$ is referred to as the critical transmission range for connectivity of all nodes. In this paper, we derive the precise asymptotic distributions of $\rho_{n}$ and $\rho_{n}^{\prime}$.
\end{abstract}

\section{INTRODUCTION}

A wireless ad hoc network is a collection of radio devices (transceivers) located in a geographic region. Each node is equipped with an omnidirectional antenna and has limited transmission power. A communication session is established either through a single-hop radio transmission if the communication parties are close enough, or through relaying by intermediate devices otherwise. Because of the no need for a fixed infrastructure, wireless ad hoc networks can be flexibly deployed at low cost for varying missions such as decision making in the battlefield, emergency disaster relief and environmental monitoring. In most applications, the ad hoc wireless devices are deployed in a large volume. The sheer large number of devices deployed coupled with the potential harsh environment often hinders or completely eliminates the possibility of strategic device placement, and consequently, random deployment is often the only viable option. In some other applications, the ad hoc wireless devices may be continuously in motion or be dynamically switched to on or off. For all these applications, it is natural to represent the ad hoc devices by a finite random point process over the (finite) deployment region. Correspondingly, the wireless ad hoc network is represented by a random graph.

The classic random graph model due to Erdős and Rényi (1960) [4], in which each pair of vertices are joined by an edge independently and uniformly at some probability, is not suited to accurately represent networks of short-range radio nodes due to the presence of local correlation among radio links. This motivated Gilbert (1961) [5] to propose an alternative random graph model for radio networks. Gilbert's model assumes that all devices, represented by an infinite random point process over the entire plane, have the same maximum transmission radius $r$ and two devices are joined by an edge if and only if their distance is at most $r$. For the modelling of wireless ad hoc networks which consist of finite radio nodes in a bounded geographic region, a bounded (or finite) variant of the standard Gilbert's model has been used by Gupta and Kumar (1998) [6] and others. In this variant, the random point precess representing the ad hoc devices is typically assumed to be a uniform n-point process $\mathcal{X}_{n}$ over a unit-area disk or square by proper scaling, and the wireless ad hoc network, denoted by $\mathcal{G}_{r}\left(\mathcal{X}_{n}\right)$, is exactly the $r$-disk graph over $\mathcal{X}_{n}$. To distinguish the random graph $\mathcal{G}_{r}\left(\mathcal{X}_{n}\right)$ from the classic random graph due to Erdôs and Rényi, it is referred to as a random geometric graph.

In this paper, we consider an extension to the random geometric graph $\mathcal{G}_{r}\left(\mathcal{X}_{n}\right)$ by introducing an additional assumption that all nodes are active (or available) independently with probability $p$ for some constant $0<p \leq 1$. These nodes are referred to as Bernoulli nodes since the availability of these nodes follows the Bernoulli model with parameter $p$. Two natural random geometric graphs can be defined over wireless ad hoc networks of Bernoulli nodes. The first one, denoted by $\mathcal{H}_{r}\left(\mathcal{X}_{n}\right)$, is the $r$-disk graph over the active nodes. In other words, $\mathcal{H}_{r}\left(\mathcal{X}_{n}\right)$ is the subgraph of $\mathcal{G}_{r}\left(\mathcal{X}_{n}\right)$ induced by the active nodes. The second one, denoted by $\mathcal{H}_{r}^{\prime}\left(\mathcal{X}_{n}\right)$, is the subgraph of $\mathcal{G}_{r}\left(\mathcal{X}_{n}\right)$ which consists of all edges of $\mathcal{G}_{r}\left(\mathcal{X}_{n}\right)$ incident to at least one active node. In other words, $\mathcal{H}_{r}^{\prime}\left(\mathcal{X}_{n}\right)$ can be obtained from $\mathcal{G}_{r}\left(\mathcal{X}_{n}\right)$ by removing all edges whose endpoints are both inactive nodes. Let $\rho_{n}$ denote the random variable which is the smallest $r$ such that the graph $\mathcal{H}_{r}\left(\mathcal{X}_{n}\right)$ is connected, and $\rho_{n}^{\prime}$ denote the random variable which is the smallest $r$ such that the graph $\mathcal{H}_{r}^{\prime}\left(\mathcal{X}_{n}\right)$ is connected. $\rho_{n}$ is referred to as the critical transmission ranges for connectivity of active nodes, and $\rho_{n}^{\prime}$ is referred to as the critical transmission ranges for connectivity of all nodes. In this paper, we shall derive the precise asymptotic distributions of $\rho_{n}$ and $\rho_{n}^{\prime}$ when the deployment region is a unit-area disk. The same asymptotic distributions hold when the deployment region is a unit-area square, and can be obtained in the similar but slightly simpler approach.

The Bernoulli node model can be used to reinterpret various network design problems arising from wireless ad hoc networks, and the probability distributions derived in this paper offered a unified and complete solution to these problems. Below we list some which have received much interests recently:

- Fault-tolerance: In a practical wireless ad hoc network, due to either internal breakdown or harsh environment, a node may fail with some constant probability $p$. The failure nodes will not take part in routing/relaying and thus may affect the connectivity of the network formed by the "good" nodes. By modelling all nodes with the Bernoulli model with parameter $p$, the network connectivity can tolerate such random failures if and only if the graph $\mathcal{H}_{r}\left(\mathcal{X}_{n}\right)$ is connected.

- Randomized construction of virtual backbone: A virtual backbone is a connected dominating set of the network topology. A node is said to be a dominator if it belongs to the virtual back- 
bone and a dominatee otherwise. In a randomized construction of virtual backbone, each node volunteers to be a dominator independently and uniformly with constant probability $p$. By modelling all nodes with the Bernoulli model with parameter $p$, the dominators form a virtual backbone if and only if the graph $\mathcal{H}_{r}^{\prime}\left(\mathcal{X}_{n}\right)$ is connected.

- Randomized broadcast routing: In a randomized broadcast routing, each node volunteers to relay a broadcast message independently and uniformly with constant probability $p$. By modelling all nodes with the Bernoulli model with parameter $p$, the broadcast message can reach all nodes if and only if the graph $\mathcal{H}_{r}^{\prime}\left(\mathcal{X}_{n}\right)$ is connected.

- Randomized wake/sleep management: For the purpose of energy conservation, a randomized awake/sleep management lets each node wake independently and uniformly with some constant probability $p$. To maintain the network connectivity, all awake nodes should form a connected graph and every asleep node must be adjacent to some awake node. By modelling all nodes with the Bernoulli model with parameter $p$, the network connectivity is maintained if and only if the graph $\mathcal{H}_{r}^{\prime}\left(\mathcal{X}_{n}\right)$ is connected.

In what follows, $\|x\|$ is the Euclidean norm of a point $x \in \mathbb{R}^{2} .|A|$ and $\operatorname{diam}(A)$ are shorthand for 2-dimensional Lebesgue measure (or area) and diameter respectively of a measurable set $A \subset \mathbb{R}^{2}$. All integrals considered will be Lebesgue integrals. The topological boundary of a set $A \subset \mathbb{R}^{2}$ is denoted by $\partial A$. The disk of radius $r$ centered at $x$ is denoted by $D(x, r)$. The special unit-area disk centered at the origin is denoted by $\Omega$. For any set $S$ and positive integer $k$, the $k$-fold Cartesian product of $S$ is denoted by $S^{k}$. An event is said to be asymptotic almost sure (abbreviated by a.a.s.) if it occurs with a probability converges to one as $n \rightarrow \infty$. An event is said to be asymptotic almost rare (abbreviated by a.a.r.) if it occurs with a probability converges to zero as $n \rightarrow \infty$. The symbols $O, o, \sim$ always refer to the limit $n \rightarrow \infty$. To avoid trivialities, we tacitly assume $n$ to be sufficiently large if necessary. For simplicity of notation, the dependence of sets and random variables on $n$ will be frequently suppressed.

The remaining of this paper is organized as follows. In section II, we describe some related works on random geometric graphs. In section III, we present several useful geometric results and integrals. In Section IV, we derive the asymptotic distributions of $\rho_{n}$ and $\rho_{n}^{\prime}$. Finally we summarize this paper in Section V.

\section{RELATED WORKS}

The connectivity of the random geometric graph $\mathcal{G}_{r}\left(\mathcal{X}_{n}\right)$ has been studied by Dette and Henze (1989) [3] and Penrose (1997) [10]. For $r=\sqrt{\frac{\ln n+\xi}{\pi n}}$ where $\xi$ is a constant, Dette and Henze (1989) [3] showed that the graph $\mathcal{G}_{r}\left(\mathcal{X}_{n}\right)$ has no isolated nodes with probability $\exp \left(-e^{-\xi}\right)$ asymptotically. Eight years later, Penrose (1997) [10] established that if a random geometric graph $\mathcal{G}_{r}\left(\mathcal{X}_{n}\right)$ has no isolated nodes, then it is almost surely connected. These results are the exact analogue of the counterpart in classic random graphs. However, as pointed out by Bollobás (2001) [1], we should not be misled by the remembrance: the proof for the random geometric graph is much harder.

Other earlier simulation studies and/or loose analytical results on asymptotic critical transmission radius for connectivity of $\mathcal{G}_{r}\left(\mathcal{X}_{n}\right)$ can be found in [2], [8], [9], [12], [13].
Recently, Yi et al. (2003) [14] studied the probability distributions of the number of isolated nodes in $\mathcal{H}_{r}\left(\mathcal{X}_{n}\right)$ and $\mathcal{H}_{r}^{\prime}\left(\mathcal{X}_{n}\right)$ respectively.

Theorem 1: Let $r=\sqrt{\frac{\ln n+\xi}{\pi p n}}$. Then the total number of isolated nodes in $\mathcal{H}_{r}\left(\mathcal{X}_{n}\right)$ is asymptotically Poisson with mean $p e^{-\xi}$, and the total number of isolated nodes in $\mathcal{H}_{r}^{\prime}\left(\mathcal{X}_{n}\right)$ is also asymptotically Poisson with mean $e^{-\xi}$.

Let $\sigma_{n}$ denote the random variable which is the smallest $r$ such that the graph $\mathcal{H}_{r}\left(\mathcal{X}_{n}\right)$ has no isolated nodes, and $\sigma_{n}^{\prime}$ denote the random variable which is the smallest $r$ such that the graph $\mathcal{H}_{r}^{\prime}\left(\mathcal{X}_{n}\right)$ has no isolated nodes. The following theorem on the asymptotic distributions of $\sigma_{n}$ and $\sigma_{n}^{\prime}$ is an immediate consequence of Theorem 1 .

$$
\begin{aligned}
& \text { Theorem 2: Let } r_{n}(\xi)=\sqrt{\frac{\ln n+\xi}{\pi p n}} \text { for some constant } \xi \text {. Then } \\
& \qquad \begin{array}{r}
\operatorname{Pr}\left(\sigma_{n} \leq r_{n}(\xi)\right) \sim \exp \left(-p e^{-\xi}\right) \\
\operatorname{Pr}\left(\sigma_{n}^{\prime} \leq r_{n}(\xi)\right) \sim \exp \left(-e^{-\xi}\right) \\
\text { III. GEOMETRY OF DISKS }
\end{array}
\end{aligned}
$$

\section{GeOMETRY OF Disks}

The results in this section are purely geometric, with no probabilistic content. Let $r$ be the transmission radius of the nodes. For any finite set of nodes $\left\{x_{1}, \cdots, x_{k}\right\}$ in $\Omega$, we use $G_{r}\left(x_{1}, \cdots, x_{k}\right)$ to denote the graph over $\left\{x_{1}, \cdots, x_{k}\right\}$ in which there is an edge between two nodes if and only if their Euclidean distance is at most $r$. For any positive integers $k$ and $m$ with $1 \leq m \leq k$, let $C_{k m}$ denote the set of $\left(x_{1}, \cdots, x_{k}\right) \in \Omega^{k}$ satisfying that $G_{2 r}\left(x_{1}, \cdots, x_{k}\right)$ has exactly $m$ connected components.

We partition the unit-area disk $\Omega$ into three regions, $\Omega(0), \Omega(1)$ and $\Omega(2)$ as shown in Fig. 1: $\Omega(0)$ is the disk of radius $1 / \sqrt{\pi}-r$ centered at the origin; $\Omega(1)$ is the annulus of radii $1 / \sqrt{\pi}-r$ and $\sqrt{1 / \pi-r^{2}}$ centered at the origin; and $\Omega(2)$ is the annulus of radii $\sqrt{1 / \pi-r^{2}}$ and $1 / \sqrt{\pi}$ centered at the origin. Then,

$$
\begin{aligned}
& |\Omega(0)|=(1-\sqrt{\pi} r)^{2}, \\
& |\Omega(1)|=2 \pi r(1 / \sqrt{\pi}-r), \\
& |\Omega(2)|=\pi r^{2} .
\end{aligned}
$$

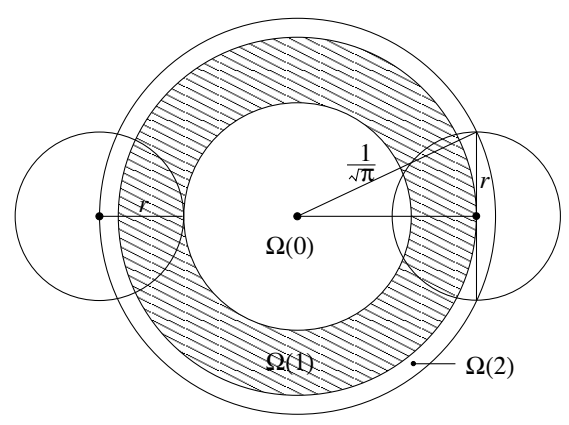

Fig. 1. The partition of the unit-area disk $\Omega$.

For any set $S \subseteq \Omega$ and $r>0$, the $r$-neighborhood of $S$ is the set $\bigcup_{x \in S} D(x, r) \cap \Omega$. We use $\nu_{r}(S)$ to denote the area of the $r$ neighborhood of $S$, and sometimes by slightly abusing the notation, to denote the $r$-neighborhood of $S$ itself. Obviously, for any $x \in \Omega$, $\nu_{r}(x) \geq \pi r^{2} / 3$. If $x \in \Omega(0), \nu_{r}(x)=\pi r^{2}$. If $x \in \Omega(1)$, then it was proved in [14] that

$$
\nu_{r}(x) \geq \frac{\pi r^{2}}{2}+\left(\frac{1}{\sqrt{\pi}}-\|x\|\right) r .
$$


The next lemma proved in [14] gives a lower bound on the area of the $r$-neighborhood of more than one nodes, and implies the following corollary whose proof was also given in [14].

Lemma 3: Assume that

$$
r \leq \frac{1 / \sqrt{\pi}}{12 / \pi+\pi / 12} \approx 0.245 / \sqrt{\pi} .
$$

Let $x_{1}, \cdots, x_{k}$ be a sequence of $k \geq 2$ nodes in $\Omega$ such that $x_{1}$ has the largest norm, and $\left\|x_{i}-x_{j}\right\| \leq 2 r$ if and only if $|i-j| \leq 1$. Then

$$
\nu_{r}\left(x_{1}, \cdots, x_{k}\right) \geq \nu_{r}\left(x_{1}\right)+\frac{\pi}{12} r \sum_{i=1}^{k-1}\left\|x_{i+1}-x_{i}\right\| .
$$

Corollary 4: Assume that

$$
r \leq \frac{1 / \sqrt{\pi}}{12 / \pi+\pi / 12} \approx 0.245 / \sqrt{\pi} .
$$

Then for any $\left(x_{1}, \cdots, x_{k}\right) \in C_{k 1}$ with $x_{1}$ being the one of the largest norm among $x_{1}, \cdots, x_{k}$,

$$
\nu_{r}\left(x_{1}, \cdots, x_{k}\right) \geq \nu_{r}\left(x_{1}\right)+\frac{\pi}{12} r \max _{2 \leq i \leq k}\left\|x_{i}-x_{1}\right\| .
$$

The following two lemmas proved in [14] will also be used in this paper.

Lemma 5: For any $z \in\left[0, \frac{1}{2}\right], e^{-z-z^{2}} \leq 1-z \leq e^{-z}$.

Lemma 6: Let $r=\sqrt{\frac{\ln n+\xi}{\pi p n}}$ for some constant $\xi$. Then for any fixed integer $k \geq 1$,

$$
\begin{aligned}
n^{k} \int_{\Omega^{k}} e^{-n p \nu_{r}\left(x_{1}, x_{2}, \cdots, x_{k}\right)} \prod_{i=1}^{k} d x_{i} & \sim e^{-k \xi}, \\
n^{k} \int_{\Omega^{k}}\left(1-p \nu_{r}\left(x_{1}, x_{2}, \cdots, x_{k}\right)\right)^{n-k} \prod_{i=1}^{k} d x_{i} & \sim e^{-k \xi} .
\end{aligned}
$$

In the remaining of this section, we introduce two new technical lemmas that will be used later in this paper. Due to limitation on paper length, proofs will not be given here.

Lemma 7: Assume that $r \leq 0.05 / \sqrt{\pi}$. Then for any $A \subset \Omega$ with diameter $\ell \leq 0.425 / \sqrt{\pi}$,

$$
\nu_{r}(A) \geq \nu_{r}(x)+|A|+\frac{1}{6} \min \left\{\pi r^{2}, \ell r\right\},
$$

where $x$ is a point in $A$ with the largest norm.

For any set $A \subseteq \Omega$, the closure of each connected component of the set $\Omega \backslash A$ referred to as a hole component of $A$. An illustration of the hole components is given in Fig. 2 . The next lemma gives a property of a closed connected set and its complementary components.

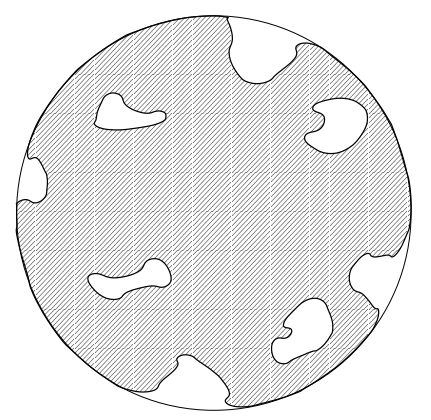

Fig. 2. The hole components (indicated by the white regions) of a set $A$ (indicated by the shaded region).
Lemma 8: Let $A \subseteq \Omega$ be a closed connected set, and $\ell=$ $\min (\operatorname{diam}(A), 0.5 / \sqrt{\pi})$. If there is a hole component of $A$ whose diameter is at least $\ell$, then the boundary of $A$ contains a curve which is inside the interior of $\Omega$ and whose diameter is at least $\ell$.

\section{CRitical TRAnsmission Ranges For Connectivity}

The main result of this section is the following theorem.

Theorem 9: The two events $\rho_{n}=\sigma_{n}$ and $\rho_{n}^{\prime}=\sigma_{n}^{\prime}$ are both a.a.s..

Theorem 9 implies that $\rho_{n}$ has the same asymptotic distribution as $\sigma_{n}$, and $\rho_{n}^{\prime}$ has the same asymptotic distribution as $\sigma_{n}^{\prime}$. Thus, together with Theorem 2, the precise asymptotic distributions of $\rho_{n}$ and $\rho_{n}^{\prime}$ are derived.

To prove Theorem 9, we introduce three events. Throughout of this section, we let $r_{n}(\xi)=\sqrt{\frac{\ln n+\xi}{\pi p n}}$. For any two real constants $\alpha<\beta$, let $K_{n}(\alpha, \beta)$ be the event that some active node of $\mathcal{X}_{n}$ is isolated in $\mathcal{H}_{r_{n}(\alpha)}\left(\mathcal{X}_{n}\right)$ but has at least two (active) neighbors in $\mathcal{H}_{r_{n}(\beta)}\left(\mathcal{X}_{n}\right)$. In other words, $K_{n}(\alpha, \beta)$ is the event that for some active node $X$ of $\mathcal{X}_{n}$, the disk $D\left(X, r_{n}(\alpha)\right)$ contains no other active nodes of $\mathcal{X}_{n}$ except $X$ and the annulus $D\left(X, r_{n}(\beta)\right) \backslash D\left(X, r_{n}(\alpha)\right)$ contains at least two active nodes of $\mathcal{X}_{n}$. For any positive constant $\mu$ and any real constant $\xi$, let $E_{n}(\mu ; \xi)$ be the event that $\mathcal{H}_{r_{n}(\xi)}\left(\mathcal{X}_{n}\right)$ has a connected component of at least two nodes whose diameter is at most $\mu r_{n}(\xi)$, and let $F_{n}(\mu ; \xi)$ be the event that $\mathcal{H}_{r_{n}(\xi)}\left(\mathcal{X}_{n}\right)$ has at least two components whose diameters are both more than $\mu r_{n}(\xi)$. The proof of Theorem 9 shall be based on the following three lemmas and is similar to but more involved than that in [11].

Lemma 10: For any two real constants $\alpha<\beta$, we have

$$
\lim _{n \rightarrow \infty} \operatorname{Pr}\left(K_{n}(\alpha, \beta)\right) \leq p(\beta-\alpha)^{2} e^{-\alpha} .
$$

Lemma 11: For any $\mu>0$ and any $\xi, E_{n}(\mu ; \xi)$ is a.a.r.

Lemma 12: For $\mu=32 \sqrt{2}+0.5 \approx 45.755$ and any $\xi, F_{n}(\mu ; \xi)$ is a.a.r..

The proofs of these three lemmas are quite lengthy and involves intensive probabilistic arguments and calculations. In order to gain a big picture of their relevance to Theorem 9, we postpone their proofs and shall first give the proof of Theorem 9 using these three lemmas.

Proof: [Proof of Theorem 9] We first show that the event $\rho_{n}=$ $\sigma_{n}$ is a.a.s.. It is obvious that $\sigma_{n} \leq \rho_{n}$. We prove that $\sigma_{n}<\rho_{n}$ is an a.a.r. event using a "squeezing" argument as in [11]. Let $\varepsilon>0$. Choose a sequence of strictly increasing numbers $\xi_{1}, \xi_{2}, \cdots, \xi_{k}$ such that

$$
\begin{aligned}
e^{-p e^{-\xi_{1}}} & <\varepsilon, \\
e^{-p e^{-\xi_{k}}} & >1-\varepsilon, \\
p e^{-\xi_{1}} \sum_{i=1}^{k-1}\left(\xi_{i+1}-\xi_{i}\right)^{2} & <\varepsilon .
\end{aligned}
$$

Let $\mu=32 \sqrt{2}+0.5 \approx 45.755$. We claim that the event $\sigma_{n}<\rho_{n}$ implies the event

$$
\begin{aligned}
& \left(\sigma_{n} \leq r_{n}\left(\xi_{1}\right)\right) \cup\left(\sigma_{n}>r_{n}\left(\xi_{k}\right)\right) \cup\left(\bigcup_{i=1}^{k} E_{n}\left(\mu ; \xi_{i}\right)\right) \\
& \cup\left(\bigcup_{i=1}^{k} F_{n}\left(\mu ; \xi_{i}\right)\right) \cup\left(\bigcup_{i=1}^{k-1} K_{n}\left(\xi_{i}, \xi_{i+1}\right)\right)
\end{aligned}
$$


Suppose that the event $\sigma_{n}<\rho_{n}$ occurs. The claim holds if either $\sigma_{n} \leq r_{n}\left(\xi_{1}\right)$ or $\sigma_{n}>r_{n}\left(\xi_{k}\right)$. So we assume that $r_{n}\left(\xi_{1}\right)<\sigma_{n} \leq$ $r_{n}\left(\xi_{k}\right)$. We consider two cases.

Case 1: $\sigma_{n} \leq r_{n}\left(\xi_{i}\right)<\rho_{n}$ for some $1<i \leq k$. Then the graph $\mathcal{H}_{r_{n}\left(\xi_{i}\right)}\left(\mathcal{X}_{n}\right)$ contains no isolated nodes but is disconnected. So $\mathcal{H}_{r_{n}(\xi)}\left(\mathcal{X}_{n}\right)$ contains at least two connected components, none of which is a singleton. According to whether the smallest of the diameters of these connected components is less than, or no less than, $\mu r_{n}\left(\xi_{i}\right)$, this implies that $E_{n}\left(\mu ; \xi_{i}\right)$ or $F_{n}\left(\mu ; \xi_{i}\right)$ occurs. Thus, our claim holds in this case.

Case 2: $r_{n}\left(\xi_{i}\right)<\sigma_{n}<\rho_{n} \leq r_{n}\left(\xi_{i+1}\right)$ for some $1 \leq i<k$. Let $X Y$ be the (unique) longest edge of the minimum spanning tree of the active nodes of $\mathcal{X}_{n}$, i.e., $\|X Y\|=\rho_{n}$. Then both $X$ and $Y$ have at least two neighbors in $\mathcal{H}_{r_{n}\left(\xi_{i+1}\right)}\left(\mathcal{X}_{n}\right)$. Indeed, $X$ has one active neighbor at a distance of at most $\sigma_{n}$, and another neighbor $Y$ at the distance $\rho_{n} \leq r_{n}\left(\xi_{i+1}\right)$. The same argument holds for $Y$. If either $X$ or $Y$ is isolated in $\mathcal{H}_{r_{n}\left(\xi_{i}\right)}\left(\mathcal{X}_{n}\right)$, then the event $K_{n}\left(\xi_{i}, \xi_{i+1}\right)$ occurs and thus our claim holds. So we assume that neither $X$ nor $Y$ is isolated in $\mathcal{H}_{r_{n}\left(\xi_{i}\right)}\left(\mathcal{X}_{n}\right)$. Since $\rho_{n}>r_{n}\left(\xi_{i}\right), X$ and $Y$ must belong to different connected components of $\mathcal{H}_{r_{n}\left(\xi_{i}\right)}\left(\mathcal{X}_{n}\right)$ and neither of these two components is a singleton. According to whether the diameters of these two connected components are both more than $\mu r_{n}\left(\xi_{i}\right)$ or not, this implies that $F_{n}\left(\mu ; \xi_{i}\right)$ or $E_{n}\left(\mu ; \xi_{i}\right)$ occurs. So our claim also holds in this case.

By Lemma 11 and Lemma $12, E_{n}\left(\mu ; \xi_{i}\right)$ and $F_{n}\left(\mu ; \xi_{i}\right)$ are a.a.r. events for each $1<i \leq k$. By Theorem 2 ,

$$
\begin{aligned}
& \lim \operatorname{Pr}\left(\sigma_{n} \leq r_{n}\left(\xi_{1}\right)\right)=e^{-p e^{-\xi_{1}}}<\varepsilon, \\
& \lim \operatorname{Pr}\left(\sigma_{n}>r_{n}\left(\xi_{k}\right)\right)=1-e^{-p e^{-\xi_{k}}}<\varepsilon .
\end{aligned}
$$

By Lemma 10,

$$
\begin{aligned}
& \lim \operatorname{Pr}\left(\bigcup_{i=1}^{k-1} K_{n}\left(\xi_{i}, \xi_{i+1}\right)\right) \leq \sum_{i=1}^{k-1} \lim \operatorname{Pr}\left(K_{n}\left(\xi_{i}, \xi_{i+1}\right)\right) \\
& \leq \sum_{i=1}^{k-1} p e^{-\xi_{i}}\left(\xi_{i+1}-\xi_{i}\right)^{2} \leq p e^{-\xi_{1}} \sum_{i=1}^{k-1}\left(\xi_{i+1}-\xi_{i}\right)^{2}<\varepsilon .
\end{aligned}
$$

Therefore,

$$
\lim \operatorname{Pr}\left(\sigma_{n}<\rho_{n}\right)<3 \varepsilon
$$

Since $\varepsilon>0$ is arbitrary, the event $\sigma_{n}<\rho_{n}$ is a.a.r.. Thus $\rho_{n}=\sigma_{n}$ is a.a.s..

Next, we show that the event $\rho_{n}^{\prime}=\sigma_{n}^{\prime}$ is a.a.s.. Note that $\rho_{n}^{\prime}=$ $\max \left\{\rho_{n}, \sigma_{n}^{\prime}\right\}$. Since the event $\rho_{n}=\sigma_{n}$ is a.a.s., the event $\rho_{n}^{\prime}=$ $\max \left\{\sigma_{n}, \sigma_{n}^{\prime}\right\}$ is also a.a.s. As $\sigma_{n} \leq \sigma_{n}^{\prime}, \max \left\{\sigma_{n}, \sigma_{n}^{\prime}\right\}=\sigma_{n}^{\prime}$. Therefore, the event $\rho_{n}^{\prime}=\sigma_{n}^{\prime}$ is a.a.s..

The remaining of this section is devoted to the proofs of Lemma 10, Lemma 11, and Lemma 12. Among them, the proof of Lemma 10 is the simplest and hence is given first.

Proof: [Proof of Lemma 10]For any $3 \leq i \leq n$, let $K_{n}^{i}(\alpha, \beta)$ be the event that $\left\{X_{1}, \cdots, X_{i}\right\}$ is the set of active nodes, the disk $D\left(X_{1}, r_{n}(\alpha)\right)$ contains no nodes in $\left\{X_{2}, \cdots, X_{i}\right\}$ and the annulus $D\left(X_{1}, r_{n}(\beta)\right) \backslash D\left(X_{1}, r_{n}(\alpha)\right)$ contains at least two nodes in $\left\{X_{2}, \cdots, X_{i}\right\}$. By symmetry,

$$
\operatorname{Pr}\left(K_{n}(\alpha, \beta)\right) \leq \sum_{i=3}^{n}\left(\begin{array}{c}
n \\
i
\end{array}\right) i \operatorname{Pr}\left(K_{n}^{i}(\alpha, \beta)\right)
$$

For any $x \in \Omega$,

$$
\begin{aligned}
\operatorname{Pr} & \left(K_{n}^{i}(\alpha, \beta) \mid X_{1}=x\right) \\
= & p^{i} q^{n-i} \sum_{j=2}^{i-1}\left(\begin{array}{c}
i-1 \\
j
\end{array}\right)\left(\nu_{r_{n}(\beta)}(x)-\nu_{r_{n}(\alpha)}(x)\right)^{j} \\
& \cdot\left(1-\nu_{r_{n}(\beta)}(x)\right)^{i-1-j} \\
= & \left(\nu_{r_{n}(\beta)}(x)-\nu_{r_{n}(\alpha)}(x)\right)^{2} p^{i} q^{n-i} \sum_{j=2}^{i-1} \frac{(i-1)(i-2)}{j(j-1)}\left(\begin{array}{c}
i-3 \\
j-2
\end{array}\right) \\
& \cdot\left(\nu_{r_{n}(\beta)}(x)-\nu_{r_{n}(\alpha)}(x)\right)^{j-2}\left(1-\nu_{r_{n}(\beta)}(x)\right)^{i-1-j} \\
\leq & \left(\pi r_{n}(\beta)^{2}-\pi r_{n}(\alpha)^{2}\right)^{2} p^{i} q^{n-i}(i-1)(i-2) \sum_{j=2}^{i-1}\left(\begin{array}{c}
i-3 \\
j-2
\end{array}\right) \\
& \cdot\left(\nu_{r_{n}(\beta)}(x)-\nu_{r_{n}(\alpha)}(x)\right)^{j-2}\left(1-\nu_{r_{n}(\beta)}(x)\right)^{i-1-j} \\
= & \left(\frac{\beta-\alpha}{n}\right)^{2} p^{i-2} q^{n-i}(i-1)(i-2)\left(1-\nu_{r_{n}(\alpha)}(x)\right)^{i-3} .
\end{aligned}
$$

Hence,

$$
\begin{aligned}
\operatorname{Pr} & \left(K_{n}^{i}(\alpha, \beta)\right)=\int_{\Omega} \operatorname{Pr}\left(K_{n}^{i}(\alpha, \beta) \mid X_{1}=x\right) d x \\
\leq & \left(\frac{\beta-\alpha}{n}\right)^{2} p^{i-2} q^{n-i}(i-1)(i-2) \\
& \cdot \int_{\Omega}\left(1-\nu_{r_{n}(\alpha)}(x)\right)^{i-3} d x .
\end{aligned}
$$

Therefore,

$$
\begin{aligned}
\operatorname{Pr}\left(K_{n}(\alpha, \beta)\right) \leq \sum_{i=3}^{n}\left(\begin{array}{c}
n \\
i
\end{array}\right) i \operatorname{Pr}\left(K_{n}^{i}(\alpha, \beta)\right) \\
\leq\left(\frac{\beta-\alpha}{n}\right)^{2} \sum_{i=3}^{n}\left(\begin{array}{c}
n \\
i
\end{array}\right) i(i-1)(i-2) p^{i-2} q^{n-i} \\
\quad \cdot \int_{\Omega}\left(1-\nu_{r_{n}(\alpha)}(x)\right)^{i-3} d x \\
=p\left(\frac{\beta-\alpha}{n}\right)^{2} n(n-1)(n-2) \int_{\Omega}\left(1-p \nu_{r_{n}(\alpha)}(x)\right)^{n} d x \\
\leq p(\beta-\alpha)^{2}\left(n \int_{\Omega}\left(1-p \nu_{r_{n}(\alpha)}(x)\right)^{n} d x\right) \\
\sim p(\beta-\alpha)^{2} e^{-\alpha},
\end{aligned}
$$

where the last asymptotic equality follows from Lemma 6 .

The proofs of Lemma 11 and Lemma 12 shall use a tessellation technique. Given a positive number $a$, a (square) $a$-tessellation divides the plane into squares of side $a$ with the origin being a corner point of a square. Each square in the tessellation, also referred to as a cell, is said to be full if it is entirely contained in $\Omega$. For any set $\tau$ of squares, let $A_{\tau}$ be the union of the squares in $\tau$. For any set $\tau$ of squares and any point $x$, let $A_{\tau x}$ be intersection of $A_{\tau}$ and the disk $D(o,\|x\|)$. For any set $\tau$ of squares, let $G_{\tau}$ be the graph over $\tau$ in which there is an edge between two squares if and only if the two squares share a side or a corner point. A set $\tau$ of squares is said to be $*$-connected if the graph $G_{\tau}$ is connected. Let $\mathcal{T}_{a, i}^{*}$ denote the collection of $*_{-}$ connected sets which consists of $i$ full squares in the $a$-tessellation. By the argument of Peierls [7], there exists two positive constant $c$ and $\gamma$ such that the cardinality of $\mathcal{T}_{a, i}{ }^{*}$ is upper-bounded by $c e^{\gamma i} / a^{2}$.

Proof: [Proof of Lemma 12]Fix $\mu=32 \sqrt{2}+0.5 \approx 45.755$ and a constant $\xi$. We also write $r_{n}(\xi)$ simply by $r$. Consider an $(r / 8)$-tessellation of the plane. Let $F^{\prime}$ denote the event that no active nodes fall in some set of at least $k=256 *$-connected full cells. We claim that $F_{n}(\mu ; \xi) \subseteq F^{\prime}$. Suppose $F_{n}(\mu ; \xi)$ occurs. Let $U_{1}$ and $U_{2}$ are connected components of $\mathcal{H}_{r}\left(\mathcal{X}_{n}\right)$ whose diameters are more than $\mu r$. Let $S_{1}$ and $S_{2}$ be the $r / 2$-neighborhoods of $U_{1}$ and 
$U_{2}$ respectively. Then they are disjoint and both have diameter more than $\mu \mathrm{r}$. By symmetry, we assume that $\operatorname{diam}\left(S_{1}\right) \leq \operatorname{diam}\left(S_{2}\right)$. Then

$$
\operatorname{diam}\left(S_{2}\right) \geq \operatorname{diam}\left(S_{1}\right) \geq \min \left\{\operatorname{diam}\left(S_{1}\right), 0.5 / \sqrt{\pi}\right\} .
$$

By Lemma 8 , the boundary of $S_{1}$ contains a piece $C$ which is contained in the interior of $\Omega$ (except the endpoints) and whose two endpoints are separated by a distance at least $\min \left\{\operatorname{diam}\left(S_{1}\right), \frac{1}{2 \sqrt{\pi}}\right\}$. Note that for sufficiently large $n, \mu r<\frac{1}{2 \sqrt{\pi}}$ and thus the distance between the two endpoints of $C$ is greater than $\mu r$. Since $C$ is contained in the interior of $\Omega$, each point of $C$ is at a distance of exactly $r / 2$ from $U_{1}$. Now move each point of $C$ towards the origin by a distance of $r / 4$ and let $C^{\prime}$ be the resulting piece. Then the distance between $C^{\prime}$ and $U_{1}$ is between $r / 4$ and $3 r / 4$, and the distance between $C^{\prime}$ and $\partial \Omega$ is at least $r / 4$. The distance between the two endpoints of $C^{\prime}$ is greater than $(\mu-0.5) r$. Consider any cell which has non-empty intersection with $C^{\prime}$. It must be entirely contained in $\Omega$. It is also entirely contained in the $r$-neighborhood of $U_{1}$ but has no intersection with $U_{1}$. Thus, it contains no active node. Let $\tau$ denote the set of these cells which have non-empty intersection with $C^{\prime}$. Then, these cells are *-connected. In addition, the cardinality of $\tau$ is at least

$$
\frac{(\mu-0.5) r}{\sqrt{2} r / 8}=\frac{8(\mu-0.5)}{\sqrt{2}}=256 .
$$

Thus, the event $F_{n}^{\prime}$ occurs, and hence our claim holds. Consequently, it suffices to show that $F_{n}^{\prime}$ is an a.a.r. event.

For any $\tau \in \mathcal{T}_{r / 8, i}^{*}$, since $\left|A_{\tau}\right|=i(r / 8)^{2}$ we have

$$
\begin{aligned}
& \operatorname{Pr}\left(\text { no active node falls in } A_{\tau}\right)=\sum_{j=0}^{n}\left(\begin{array}{l}
n \\
j
\end{array}\right) p^{j} q^{n-j}\left(1-i(r / 8)^{2}\right)^{j} \\
& =\left(1-p i(r / 8)^{2}\right)^{n} \text {. }
\end{aligned}
$$

Thus,

$$
\begin{aligned}
& \operatorname{Pr}\left(F_{n}^{\prime}\right) \leq \sum_{i \geq k} \sum_{\tau \in \mathcal{T}_{r / 8, i}^{*}} \operatorname{Pr}\left(\text { no active node falls in } A_{\tau}\right) \\
& \leq \sum_{i \geq k} c r^{-2} e^{\gamma i}\left(1-p i(r / 8)^{2}\right)^{n} \leq c r^{-2} \sum_{i \geq k} e^{-i\left(n p(r / 8)^{2}-\gamma\right)} .
\end{aligned}
$$

Note that if $n$ is big enough,

$$
n p(r / 8)^{2}-\gamma \geq \frac{\pi}{4} n p(r / 8)^{2} .
$$

Thus, for sufficiently large $n$,

$$
\begin{aligned}
& \operatorname{Pr}\left(F_{n}^{\prime}\right) \leq c r^{-2} \sum_{i \geq k} e^{-\frac{\pi}{4} n p i(r / 8)^{2}} \\
& =c r^{-2} \frac{e^{-\frac{\pi}{4} n p k(r / 8)^{2}}}{1-e^{-\frac{\pi}{4} n p(r / 8)^{2}}} \leq c r^{-2} \frac{e^{-n \pi p r^{2}}}{1-e^{-\frac{\pi}{4} n p(r / 8)^{2}}} \\
& =c \pi p e^{-\xi} \frac{1}{(\log n+\xi)\left(1-e^{-\frac{\pi}{4} n p(r / 8)^{2}}\right)}=o(1) .
\end{aligned}
$$

Hence $F_{n}^{\prime}$ is an a.a.r. event and the lemma follows.

Finally, we give the proof of Lemma 11.

Proof: [Proof of Lemma 11]Fix a constant $\xi$, and we write $r_{n}(\xi)$ simply by $r$. Let $\mu_{0}=1 / 24$. We first prove that $E_{n}\left(\mu_{0} ; \xi\right)$ is an a.a.r. event. For any $2 \leq j \leq n$, let $S_{j}$ denote the set of $\left(x_{1}, \cdots, x_{j}\right) \in \Omega^{j}$ such that (1) the diameter of the set $\left\{x_{1}, \cdots, x_{j}\right\}$ is at most $\mu_{0} r$, (2) $x_{1}$ is the one with the largest norm among $\left\{x_{1}, \cdots, x_{j}\right\}$, (3) $x_{2}$ is the one with the largest distance from $x_{1}$ among $\left\{x_{2}, \cdots, x_{j}\right\}$, and (4) the $r$-graph over $x_{1}, \cdots, x_{j}$ is connected. For any $2 \leq j \leq i \leq n$, let $E_{i j}^{\prime}$ be the event $\left\{X_{1}, \cdots, X_{i}\right\}$ is the set of active nodes of $\mathcal{X}_{n},\left(X_{1}, \cdots, X_{j}\right) \in$
$S_{j}$, and $\left\{X_{j+1}, \cdots, X_{i}\right\}$ fall outside of the $r$-neighborhood of $\left\{X_{1}, \cdots, X_{j}\right\}$. By symmetry,

$$
\begin{aligned}
& \operatorname{Pr}\left(E_{n}\left(\mu_{0} ; \xi\right)\right) \leq \sum_{i=2}^{n} \sum_{j=2}^{i}\left(\begin{array}{c}
n \\
i
\end{array}\right)\left(\begin{array}{l}
i \\
j
\end{array}\right) j(j-1) \operatorname{Pr}\left(E_{i j}^{\prime}\right) \\
& =n(n-1) \sum_{i=2}^{n}\left(\begin{array}{c}
n-2 \\
i-2
\end{array}\right) \sum_{j=2}^{i}\left(\begin{array}{c}
i-2 \\
j-2
\end{array}\right) \operatorname{Pr}\left(E_{i j}^{\prime}\right) .
\end{aligned}
$$

By Corollary 4 for any $\left(x_{1}, \cdots, x_{j}\right) \in S_{j}$,

$$
\begin{aligned}
& \operatorname{Pr}\left(E_{i j}^{\prime} \mid\left(X_{1}, \cdots, X_{j}\right)=\left(x_{1}, \cdots, x_{j}\right)\right) \\
& =p^{i} q^{n-i}\left(1-\nu_{r}\left(x_{1}, \cdots, x_{j}\right)\right)^{i-j} \\
& \leq p^{i} q^{n-i}\left(1-\left(\nu_{r}\left(x_{1}\right)+\frac{\pi}{12} r\left\|x_{2}-x_{1}\right\|\right)\right)^{i-j},
\end{aligned}
$$

Hence,

$$
\begin{aligned}
& \operatorname{Pr}\left(E_{i j}^{\prime}\right) \\
& =\int_{S_{j}} \operatorname{Pr}\left(E_{i j}^{\prime} \mid\left(X_{1}, \cdots, X_{j}\right)=\left(x_{1}, \cdots, x_{j}\right)\right) d x_{1} \cdots d x_{j} \\
& \leq p^{i} q^{n-i} \int_{S_{j}}\left(1-\left(\nu_{r}\left(x_{1}\right)+\frac{\pi}{12} r\left\|x_{2}-x_{1}\right\|\right)\right)^{i-j} d x_{1} \cdots d x_{j} \\
& \leq p^{i} q^{n-i} \int_{\Omega} d x_{1} \\
& \quad \cdot \int_{B\left(x_{1}, \mu_{0} r\right)}\left(1-\left(\nu_{r}\left(x_{1}\right)+\frac{\pi}{12} r\left\|x_{2}-x_{1}\right\|\right)\right)^{i-j} \\
& \quad \cdot\left(\pi\left\|x_{2}-x_{1}\right\|^{2}\right)^{j-2} d x_{2} \\
& =2 \pi p^{i} q^{n-i} \int_{\Omega} d x \\
& \quad \cdot \int_{0}^{\mu_{0} r}\left(1-\left(\nu_{r}(x)+\frac{\pi}{12} r \rho\right)\right)^{i-j}\left(\pi \rho^{2}\right)^{j-2} \rho d \rho
\end{aligned}
$$

Therefore,

$$
\begin{aligned}
& \operatorname{Pr}\left(E_{n}\left(\mu_{0} ; \xi\right)\right) \\
& \leq n(n-1) \sum_{i=2}^{n}\left(\begin{array}{c}
n-2 \\
i-2
\end{array}\right) \sum_{j=2}^{i}\left(\begin{array}{c}
i-2 \\
j-2
\end{array}\right) 2 \pi p^{i} q^{n-i} \int_{\Omega} d x \\
& \quad \cdot \int_{0}^{\mu_{0} r}\left(1-\left(\nu_{r}(x)+\frac{\pi}{12} r \rho\right)\right)^{i-j}\left(\pi \rho^{2}\right)^{j-2} \rho d \rho \\
& =2 \pi p^{2} n(n-1) \int_{\Omega} d x \\
& \quad \cdot \int_{0}^{\mu_{0} r}\left(1-p \nu_{r}(x)-p\left(\frac{\pi}{12} r \rho-\pi \rho^{2}\right)\right)^{n-2} \rho d \rho \\
& \leq 2 \pi p^{2} n(n-1) \int_{\Omega} d x \int_{0}^{\mu_{0} r}\left(1-p \nu_{r}(x)-\frac{\pi}{24} p r \rho\right)^{n-2} \rho d \rho \\
& \sim 2 \pi p^{2} n(n-1) \int_{\Omega} d x \int_{0}^{\mu_{0} r}\left(1-p \nu_{r}(x)-\frac{\pi}{24} p r \rho\right)^{n} \rho d \rho \\
& \leq 2 \pi p^{2} n(n-1) \int_{\Omega} d x \int_{0}^{\mu_{0} r} e^{-n p \nu_{r}(x)-\frac{\pi}{24} p r \rho} \rho d \rho \\
& \leq 2 \pi p^{2}\left(n \int_{\Omega} e^{-n p \nu_{r}(x)} d x\right)\left(n \int_{0}^{\mu_{0} r} e^{-\frac{\pi}{24} p r \rho} \rho d \rho\right)=o(1),
\end{aligned}
$$

where the last equality follows from Lemma 6, and the fact that

$$
n \int_{0}^{\mu_{0} r} e^{-\frac{\pi}{24} \operatorname{pr} \rho} \rho d \rho=o(1) .
$$

Thus, $\operatorname{Pr}\left(E_{n}\left(\mu_{0} ; \xi\right)\right)=o(1)$.

Now we fix a constant $\mu>\mu_{0}$ and show that the event $E_{n}(\mu ; \xi) \backslash$ $E_{n}\left(\mu_{0} ; \xi\right)$ is also a.a.r.. We introduce another event $\widetilde{E}$ as follows. Let $\varepsilon=\frac{0.75}{2+576 \pi}$ and consider an $\varepsilon r$-tessellation of the plane. For any $x \in \Omega$, let $\mathcal{C}_{r}(x)$ denote the collections of subsets $\tau$ of squares in the $\varepsilon r$-tessellation satisfying that $x \in A_{\tau} \subseteq D(x,(\mu+\varepsilon) r)$ and $\operatorname{diam}\left(A_{\tau x}\right) \geq \mu_{0} r$. Then $\widetilde{E}$ is defined to be the event that there exists an active node $X$ such that all active nodes fall outside of the region 
$\nu_{(1-2 \varepsilon) r}\left(A_{\tau X}\right) \backslash A_{\tau X}$ for some $\tau \in \mathcal{C}_{r}(X)$ where $\nu_{(1-2 \varepsilon) r}\left(A_{\tau X}\right)$ represents the $(1-2 \varepsilon) r$-neighborhood of $A_{\tau X}$ by a slight abuse of the notation. We claim that

$$
E_{n}(\mu ; \xi) \backslash E_{n}\left(\mu_{0} ; \xi\right) \subseteq \widetilde{E} .
$$

Suppose $E_{n}(\mu ; \xi) \backslash E_{n}\left(\mu_{0} ; \xi\right)$ occurs. Let $U$ be a connected component of $\mathcal{H}_{r}\left(\mathcal{X}_{n}\right)$ whose diameter is more than $\mu_{0} r$ but at most $\mu r$. Let $\tau$ be the set of cells containing the points of $U$ and $X$ be the node in $U$ which has the largest norm. Clearly, $\tau \in \mathcal{C}_{r}(X)$. Since $\nu_{(1-2 \varepsilon) r}\left(A_{\tau X}\right)$ is contained in the $r$-neighborhood of $U$, no active node lies in $\nu_{(1-2 \varepsilon) r}\left(A_{\tau X}\right) \backslash A_{\tau X}$. Thus $\widetilde{E}$ also occurs. So our claim is true. Hence it suffices to show that $\widetilde{E}$ is a.a.r..

For any $1 \leq i \leq n$, let $\widetilde{E}_{i}$ be the event $\left\{X_{1}, \cdots, X_{i}\right\}$ is the set of active nodes none of which falls in $\nu_{(1-2 \varepsilon) r}\left(A_{\tau X_{1}}\right) \backslash A_{\tau X_{1}}$ for some $\tau \in \mathcal{C}_{r}(X)$. By symmetry,

$$
\operatorname{Pr}(\widetilde{E}) \leq \sum_{i=1}^{n}\left(\begin{array}{c}
n \\
i
\end{array}\right) i \operatorname{Pr}\left(E_{i}\right)=n \sum_{i=1}^{n}\left(\begin{array}{c}
n-1 \\
i-1
\end{array}\right) \operatorname{Pr}\left(E_{i}\right) .
$$

Next, we derive an upper bound on $\operatorname{Pr}\left(\widetilde{E}_{i}\right)$. Fix an $x \in \Omega$. By a straightforward area argument, the number of squares which are contained in $D(x,(\mu+\varepsilon) r)$ is bounded by a constant (independent of $x$ ). Thus the cardinality of $\mathcal{C}_{r}(x)$ is also bounded by a constant. Assume that $n$ is sufficiently large $n$ such that

$$
(1-2 \varepsilon) r \leq 0.05 / \sqrt{\pi} \text {, and }(\mu+\varepsilon) r \leq 0.425 / \sqrt{\pi} \text {. }
$$

Then by Lemma 7 for any $\tau \in \mathcal{C}_{r}(x)$,

$$
\begin{aligned}
& \left|\nu_{(1-2 \varepsilon) r}\left(A_{\tau x}\right) \backslash A_{\tau x}\right|=\nu_{(1-2 \varepsilon) r}\left(A_{\tau x}\right)-\left|A_{\tau x}\right| \\
& \geq \nu_{(1-2 \varepsilon) r}(x)+\frac{1}{6} \min \left\{\pi((1-2 \varepsilon) r)^{2}, \mu_{0} r((1-2 \varepsilon) r)\right\} \\
& =\nu_{(1-2 \varepsilon) r}(x)+\frac{1}{144}(1-2 \varepsilon) r^{2} \\
& \geq \nu_{r}(x)-\pi\left(r^{2}-((1-2 \varepsilon) r)^{2}\right)+\frac{1}{144}(1-2 \varepsilon) r^{2} \\
& \geq \nu_{r}(x)-4 \pi \varepsilon r^{2}+\frac{1}{144}(1-2 \varepsilon) r^{2} \\
& =\nu_{r}(x)+\frac{1-(2+576 \pi) \varepsilon}{144} r^{2}=\nu_{r}(x)+(r / 24)^{2} .
\end{aligned}
$$

Thus,

$$
\begin{aligned}
& \operatorname{Pr}\left(\widetilde{E}_{i} \mid X_{1}=x\right) \\
& \leq \sum_{\tau \in \mathcal{C}_{r}(x)} p^{i} q^{n-i}\left(1-\left|\left(\left(A_{\tau x}\right)_{(1-2 \varepsilon) r} \cap \Omega\right) \backslash A_{\tau x}\right|\right)^{i} \\
& \leq \Theta(1) p^{i} q^{n-i}\left(1-\left(\nu_{r}(x)+(r / 24)^{2}\right)\right)^{i} .
\end{aligned}
$$

Therefore,

$$
\begin{aligned}
& \operatorname{Pr}\left(\widetilde{E}_{i}\right)=\int_{\Omega} \operatorname{Pr}\left(\widetilde{E}_{i} \mid X_{1}=x\right) d x \\
& \leq \Theta(1) p^{i} q^{n-i} \int_{\Omega}\left(1-\left(\nu_{r}(x)+(r / 24)^{2}\right)\right)^{i} d x .
\end{aligned}
$$

So we have

$$
\begin{aligned}
& \operatorname{Pr}(\widetilde{E}) \leq n \sum_{i=1}^{n}\left(\begin{array}{c}
n-1 \\
i-1
\end{array}\right) \operatorname{Pr}\left(\widetilde{E}_{i}\right) \\
& \leq \Theta(1) n \int_{\Omega} \sum_{i=1}^{n}\left(\begin{array}{c}
n-1 \\
i-1
\end{array}\right) p^{i} q^{n-i}\left(1-\left(\nu_{r}(x)+(r / 24)^{2}\right)\right)^{i} d x \\
& =\Theta(1) n \int_{\Omega}\left(1-p\left(\nu_{r}(x)+(r / 24)^{2}\right)\right)^{n-1} d x \\
& \sim \Theta(1) n \int_{\Omega}\left(1-p\left(\nu_{r}(x)+(r / 24)^{2}\right)\right)^{n} d x \\
& \leq \Theta(1) n \int_{\Omega} e^{-n p\left(\nu_{r}(x)+(r / 24)^{2}\right)} d x
\end{aligned}
$$

$$
=\Theta(1) e^{-n p(r / 24)^{2}}\left(n \int_{\Omega} e^{-n p \nu_{r}(x)} d x\right)=o(1) .
$$

\section{CONCLUSION}

Motivated by various design issues such as fault-tolerance, randomized construction of virtual backbone, randomized broadcast routing, and randomized wake/sleep management, we study the critical transmission ranges for connectivity in wireless ad hoc works with Bernoulli nodes. Specifically, we assume that the wireless ad hoc network consists of $n$ nodes which are distributed independently and uniformly in a unit-area disk or square and are active independently with some constant probability $p$. The critical transmission range $\rho_{n}$ for connectivity of active nodes is the smallest transmission range at which the active nodes form a connected network. The critical transmission range $\rho_{n}^{\prime}$ for connectivity of all nodes is the smallest transmission range at which the active nodes form a connected network and each inactive node is adjacent to at least one active node. The critical transmission range $\sigma_{n}$ for no isolated active nodes is the smallest transmission range at which each active node is adjacent to some other active node. The critical transmission range $\sigma_{n}^{\prime}$ for connectivity of all nodes is the smallest transmission range at which each active node is adjacent to some other active node and each inactive node is adjacent to at least one active node. In this paper, we proved that the two events $\rho_{n}=\sigma_{n}$ and $\rho_{n}^{\prime}=\sigma_{n}^{\prime}$ are both a.a.s.. Based on this property, we derived the precise asymptotic distributions of $\rho_{n}$ and $\rho_{n}^{\prime}$.

As a future work, we would like to study the asymptotic critical transmission range of wireless ad hoc networks with Bernoulli links.

\section{REFERENCES}

[1] B. Bollobás, Random Graphs, Second Edition, Cambridge University Press, 2001

[2] C. Bettstetter, On the minimum node degree and connectivity of a wireless multihop network. In 3rd ACM International Symposium on Mobile Ad Hoc Networking and Computing (MobiHoc'02) (June 2002).

[3] H. Dette, and N. Henze, The limit distribution of the largest nearest neighbor link in the unit $d$-cube, J. Appl. Probab., vol. 26, pp. 67-80, 1989.

[4] P. Erdős, and A. Rényi, On the evolution of random graphs, Publ. Math. Inst. Hung. Acad. Sci. 5, 17-61, 1960.

[5] E.N. Gilbert, Random plane networks, Journal of the Society for Industrial and Applied Mathematics 9:4 (1961), 533-543.

[6] P. Gupta, and P.R. Kumar, Critical power for asymptotic connectivity in wireless networks, Stochastic Analysis, Control, Optimization and Applications: A Volume in Honor of W.H. Fleming, W. M. McEneaney, G. Yin, and Q. Zhang (Eds.) (1998).

[7] D.A. Klarner, Cell growth problems, Canadian Journal of Mathematics 19 (1967), 851-863.

[8] J. Ni, and S. Chandler, Connectivity properties of a random radio network. IEE Proceedings of Communications 141, 4 (1994).

[9] O.D. Patrick, Connectivity in ad-hoc and hybrid networks. In IEEE INFOCOM (2002).

[10] M.D. Penrose, The longest edge of the random minimal spanning tree, Ann. Appl. Probab., vol. 7, pp. 340-361, 1997.

[11] M.D. Penrose, On $k$-connectivity for a geometric random graph, Random Structures and Algorithms, vol. 15, no. 2, pp. 145-164, 1999.

[12] P. Piret, On the connectivity of radio networks. IEEE Transactions on Information Theory 37, 5 (1991), pp. 1490-1492.

[13] P. Santi, and D.M. Blough. An evaluation of connectivity in mobile wireless ad hoc networks. In Proc. IEEE DSN (2002), pp. 89-98.

[14] C.-W. Yi, P.-J. Wan, X.-Y. Li, and O. Frieder, Asymptotic distribution of the number of isolated nodes in wireless ad hoc networks with Bernoulli nodes, IEEE Wireless Communications and Networking Conference (WCNC), New Orleans, Louisiana, March 2003. 